\title{
Random Forests in the Supervised Classification of Multidimensional Images of the Tetrazolium Test
}

\author{
Davi Marcondes Rocha ${ }^{1}$, Lúcia Helena Pereira Nóbrega ${ }^{2}$, Daiane Bernardi ${ }^{3}$, Giuvane Conti ${ }^{1}$, \\ Evandro Alves Nakajima ${ }^{1}$, Magnos Fernando Ziech ${ }^{4} \&$ Claudio Leones Bazzi ${ }^{5}$ \\ ${ }^{1}$ Computer Science Department, Federal University of Technology, Paraná, Santa Helena, Paraná, Brazil \\ ${ }^{2}$ Tec. and Exact Sciences Center, Western Paraná State University, Cascavel, Paraná, Brazil \\ ${ }^{3}$ School of Agricultural Engineering, University of Campinas, Campinas, São Paulo, Brazil \\ ${ }^{4}$ Agronomy Department, Federal University of Technology, Paraná, Santa Helena, Paraná, Brazil \\ ${ }^{5}$ Computer Science Department, Federal University of Technology, Paraná, Medianeira, Paraná, Brazil \\ Correspondence: Davi Marcondes Rocha, Computer Science Department, Federal University of \\ Technology-Paraná, Santa Helena, Paraná, Brazil. E-mail: davimarcondesrocha@gmail.com
}

Received: June 17, 2019

doi:10.5539/jas.v11n15p115
Accepted: July 19, 2019 Online Published: September 15, 2019

URL: https://doi.org/10.5539/jas.v11n15p115

\begin{abstract}
The quality of the soybean seed can be influenced by several factors that may occur at any stage of production. Mechanical damage, deterioration by humidity and the damage caused by bed bugs are among such problems. The tetrazolium test is adopted by the seed industry, especially for testing soybeans, due to its accuracy, fast result, and the large amount of information it provides. Digital processing and image analysis can be used to aid the extraction and classification of standards for minimizing the subjectivity implicit in the test, thus allowing more credibility to the information. The aim of this work is testing the effectiveness of Random Forests in the supervised classification of soybean embryos images submitted to the tetrazolium test. In order to do so, we used the Trainable Weka Segmentation plugin to perform the segmentation process, and the WEKA software to evaluate the quality of the classifier model obtained. During the process, 222,646 instances among 230,388 instances were correctly classified (96.7\%), with Kappa index of 0.95 , showing the classifier excellent performance regarding the proposed dataset. The supervised classification, combined with pixel-based segmentation, proved to be efficient in extracting more coherent visual information on seed damage. Also, we conclude that the choice of image attributes, along with the algorithm used in the work, showed to be competent in the classification process of high dimensionality samples.
\end{abstract}

Keywords: seed technology, seed vigor, machine learning

\section{Introduction}

Soybeans are one of the species most in need of consideration, given the degree of collection by producers. Seed technology has as main objective to develop efficient mechanisms for the productive chain, using lots of seeds of high quality. This set of knowledge, which it is based on practical experience and scientific experimentation, begins with the work of genetic improvement and proceeds to the harvesting, processing and distribution of high-quality lots, bringing together the genetic, physiological and sanitary attributes (Marcos Filho, 2015).

The quality of soybean seed, especially in tropical regions, may be influenced by several factors, which may occur at any stage of production (field, harvest and post-harvest). Among these problems they bring, mechanically caused damages in the harvesting and processing operations, damages caused by deterioration due to humidity, arising from drought period, temperature extremes, during maturation, and fluctuations of ambient humidity conditions, and damage caused by bed bugs stand out (França Neto, Krzyzanowski, Henning, \& Costa, 2000).

The tetrazolium test has been outstanding among the quality analysis tests adopted by the seed industry, especially for soybeans, not only because of its accuracy and speed, when compared to other tests, but also because of the large number of information it provides, such as the diagnosis of possible causes of quality 
reduction (mechanical damage, deterioration by moisture, and bedbug damage), and the possibility to evaluate the quality and vigor of seed lots (França Neto \& Krzyzanowski, 2018).

Even without using expensive equipment and reagents, the accuracy of the test depends on a well-trained seed analyst, who knows all the techniques and procedures involved in the test, so the analyst's ability to recognize typical patterns of the various types of damage that can be visualized in the seeds is essential to obtain a correct diagnosis of the causes of viability loss (França Neto \& Krzyzanowski, 2018; Moore, 1985).

Computational tools that employ digital processing and image analysis to aid the extraction and classification of patterns in information that minimize or nullify the subjectivity implied in the accomplishment of some tests contribute to a greater information credibility and guarantee of the results, besides reducing the classification time of the test.

The ImageJ platform is a free distribution software, licensed under the GNU (General Public Licenses), and is used by an active community, composed by researchers from various knowledge fields. Its use allows a range of applications, from data visualization to advanced image processing and statistical analysis. Due to its extensibility, it attracts biologists and computer scientists who efficiently implement specific image processing algorithms (Schindelin, Rueden, Hiner, \& Eliceiri, 2015).

Image segmentation is generally defined as the decomposition process in non-intersecting regions, where a label is assigned to these regions (pixel set), which share certain visual characteristics. Most traditional segmentation methods are based on pixel intensity information only. However, humans use other information when performing segmentation naturally. For this reason, recently, trainable segmentation methods emerged as an important alternative to improve the accuracy of the region labeling process (Arganda-Carreras et al., 2016).

Recently, a new family of algorithms based on machine learning has been recognized as being successful for image classification, by using computational intelligence paradigms, which studies the development of inference techniques from samples. These techniques, based on mathematical models, present the ability to "learn" from the samples and generalize the knowledge generated for the whole image (Andrade, Francisco, \& Almeida, 2015).

Learning-based algorithms have been developed to obtain more accurate and reliable information as an alternative to the usual pixel-based approaches and objects. Random Forest (RF), Bagging, Boosting, Decision Tree, Artificial Neural Network, Supported Vector Machine (SVM) and K-Nearest-Neighbor are among the most commonly used learning-based algorithms. These algorithms are also known as machine learning methods, which look for the best model for the data, using a set of data with sufficient size and parameters, and decision rules created from the input data (Breiman, 2001; Akar \& Güngör, 2012).

Breiman (2001) has proposed Random Forests, that include an additional layer of randomness to the bagging procedure. Therefore, in addition to constructing each tree using a bootstrap sample different from data, Random Forests change the way classification or regression trees are built. By default, in trees, each node is divided according to the best combination of all variables. In a Random Forest, each node is divided using the best among a subset of randomly chosen predictors on that node. This classification strategy is efficient when compared to other classifiers, such as discriminant analysis, support vector machines, and neural networks, besides being robust against overfitting (Breiman, 2001; Liaw \& Wiener, 2002).

Random Forests are composed of a set of decision trees, where the prediction of the class for new values is based on a voting system, in which, after generating a large number of trees (forest), the class is chosen, based on the majority of tree votes, being formally described as $\mathrm{h}(\mathrm{x}, \Theta \mathrm{k})$, where $\mathrm{h}$ is the decision tree, $\mathrm{x}$ is the input to be sorted, and $\Theta \mathrm{k}$ is the $\mathrm{k}^{\text {th }}$ random vector sampled independently (Breiman, 2001). Hence, each tree votes for the most popular class for the $\mathrm{x}$ entry to be sorted.

These forests are obtained through a method to generate multiple versions of a predictor, known as bagging (bootstrapping aggregating) Breiman (1996), according to which the final forecast is performed by the average of predictions B (Equation 1) or by the majority vote (Equation 2) (Goldstein, Polley, \& Briggs, 2011).

$$
\hat{\mathrm{f}}_{\mathrm{av}}(\mathrm{x})=\frac{1}{\mathrm{~T}} \sum_{\mathrm{t}=1}^{\mathrm{T}} \hat{\mathrm{f}}^{\mathrm{t}}(\mathrm{x})
$$

Where, $\hat{\mathrm{f}}^{t}(\mathrm{x})$ is the function with the features to be studied; and $\mathrm{T}$ is the number of training samples.

$$
\hat{\mathrm{f}}_{\mathrm{bag}}(\mathrm{x})=\frac{1}{\mathrm{~B}} \sum_{\mathrm{b}=1}^{\mathrm{B}} \hat{\mathrm{f}}^{* \mathrm{~b}}(\mathrm{x})
$$

Where, $\hat{\mathrm{f}}^{* \mathrm{~b}}(\mathrm{x})$ is the function with the features to be studied; and $\mathrm{B}$ is the number of bootstrap samples. 
Figure 1 exemplifies the classification of an image by Random Forest, where each decision tree recursively classifies the input patch into the root node until a leaf node (class) is reached, being the patch classified according to the class that obtains most votes.

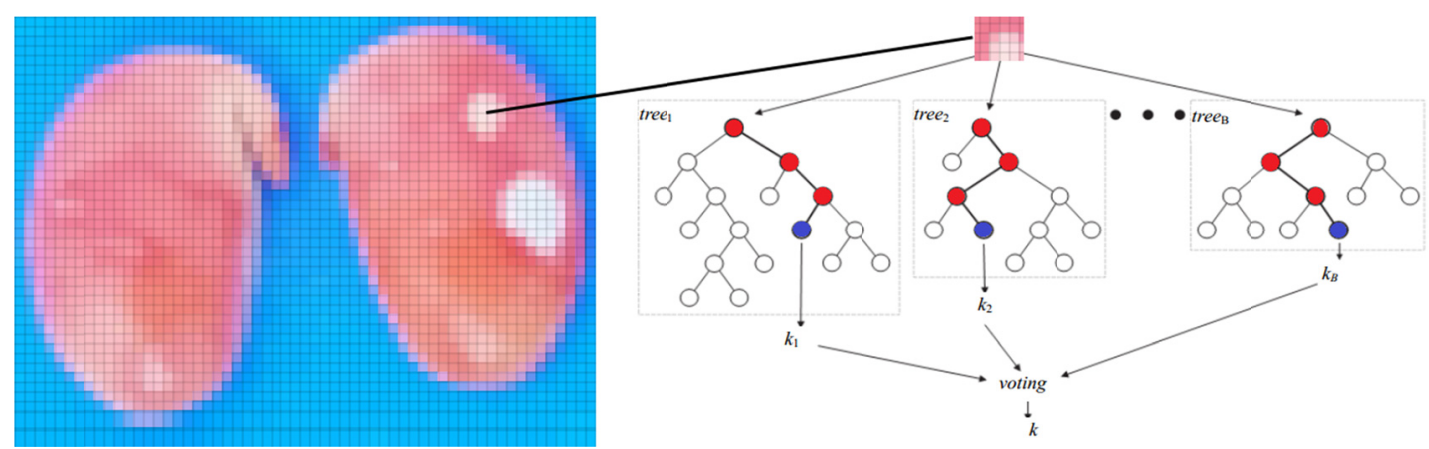

Figure 1. Example of a Random Forest classifying an input patch, and assigning it the class from the majority vote of the trees. Adapted from (Huang, Siu, \& Liu, 2015; C. Nguyen, Wang, \& H. Nguyen, 2013)

According to Akar and Güngör (2012), Random Forest is known for being one of the most efficient methods of classification and for attracting researchers from different areas of knowledge, due to its intrinsic interdisciplinary character. Therefore, this work aims to investigate the performance of the Random Forest algorithm using multidimensional images of the tetrazolium test.

\section{Materials and Methods}

This work was carried out at the Laboratory of Seed and Plant Evaluation of Western Paraná State University (UNIOESTE), Cascavel campus, and also at the Biology and Chemistry Teaching Laboratories at Federal Technological University of Paraná (UTFPR), Santa Helena campus. Soybean seeds of several cultivars from agricultural properties, as well as seeds known to be carriers of specific damages, provided by an official seed analysis laboratory in the region, were used. For performing the tetrazolium test, approximately $500 \mathrm{~g}$ of seeds were selected. The stock solution at $1.0 \%$ was prepared by mixing $10.0 \mathrm{~g}$ of tetrazolium salt in $1.0 \mathrm{~L}$ distilled water. As suggested by Association of Official Seed Analysts [AOSA] (1983) 100 seeds of each sample were used (two subsamples of 50 seeds each). The seeds were packed in moisture germination paper and kept under these conditions for 16 hours in a BOD (Biochemical Oxygen Demand) type oven at $25^{\circ} \mathrm{C}$. After this period, the seeds were put in plastic bags to receive $0.075 \%$ tetrazolium solution to stay completely submersed. Afterwards, the temperature was between 35 and $40{ }^{\circ} \mathrm{C}$ for $150-180$ minutes (2.5 to 3 hours) until the staining.

In the process of image analysis, the libraries of the FIJI software, which are a distribution of ImageJ software that adds several functionalities that facilitate the analysis of scientific image, were used. Such software was proposed as a productive collaboration platform between Computer Science researchers and Biology research groups (Schindelin et al., 2012).

To perform the segmentation of the images, the Trainable Weka Segmentation (TWS) plug-in was employed, since it is integrated with the FIJI software. This plug-in works as a link between machine learning fields and digital image processing, providing the framework needed to use and compare classifiers that perform image segmentation. It combines a collection of machine learning algorithms with one set of image characteristics, to produce pixel-based segmentations. The TWS provides a set of methods for extracting statistical properties from an image, based on pixel samples, and then, from this information, segment the rest of the pixels. Waikato Environment for Knowledge Analysis (WEKA) is open source software. It consists of a range of machine learning algorithms for data mining, which includes tools for: data preprocessing, classification, regression, clustering, association and visualization rules. All WEKA classification, regression and clustering algorithms can be used by the TWS (Hall, Frank, Holmes, Pfahringer, Reutemann, \& Witten, 2009; Arganda-Carreras, Cardona, Kaynig, Rueden, \& Schindelin, 2011; Arganda-Carreras et al., 2016).

The acquisition of the tetrazolium test images was performed by using a Sony HX200V 18.2 Mega Pixels camera, with Charge-Coupled Device (CMD), Exmor $\mathrm{R}^{\mathrm{TM}}$ CMOS sensor, without flash. The images were preprocessed (enhancement and restoration operations) to improve information or suppress the irrelevant ones. The purpose of the process was to facilitate the subsequent operations in the search for better results (Awcock \& 
Thomas, 1996). The image generated for replication I has resolution of 2304 pixels wide, 1329 pixels high, in PNG format. Replication II has a resolution of 2307 pixels wide, and 1350 pixels high, in PNG format.

The characteristics of analyzed image are color in HSB pattern and Entropy with $r_{\max }=8$ and $r_{\min }=1$. The used classifier was the FastRandomForest beginning with 200 trees and two random characteristics per knot. FastRandomForest is a multitasking reimplementation of Random Forest, created by Fran Supek, which optimizes speed and memory usage (Arganda-Carreras et al., 2016). This process aims at extracting the characteristics of image used in the system and building the decision trees, based on the characteristic vector constructed by the previous process.

After training the classifier, it is important to evaluate its ability to perform generalizations, that is, its performance with a new set of data with the same features. For this purpose, the cross-validation technique with n-fold (Equation 3) was used (Geisser, 1993).

$$
\mathrm{VC}_{(\mathrm{n})}=\frac{1}{\mathrm{n}} \sum_{\mathrm{i}=1}^{\mathrm{n}} \mathrm{E}_{\mathrm{y}_{\mathrm{i}}, \hat{\mathrm{y}}_{\mathrm{i}}}
$$

Where, $\mathrm{n}$ is the validation data number; $\mathrm{E}_{\mathrm{y}_{\mathrm{i}}, \hat{\mathrm{y}}_{\mathrm{i}}}$ is the residue from the difference between the actual output value and the predicted value.

This technique consists of stratifying the database into $\mathrm{n}$ subsets (folds), in which $\mathrm{n}-1$ are used in training, and one validates the model. This process is repeated $\mathrm{n}$ times, so that each stratification is used once as a set of tests for model validation. In each training, the classification error of the subsets is calculated, and the final result of this process is the average accuracy of the classifier in the $\mathrm{n}$ tests. In this way, an estimation of the classifier quality is obtained, allowing the analysis to be performed (Dias, Sanches, \& Alves, \& Nogueira 2012).

To evaluate the performance of the classifier on the dataset, the WEKA software was used, and the Cross-validation 10 folds method was applied to the data.

The confusion matrix was generated (Figure 2), which shows the samples that were not correctly classified, based on the reference classes. From this matrix, the following Kappa (Cohen, 1960) (Equation 4), Precision (Equation 5), Sensitivity (Equation 6), and Accuracy (Equation 7) indices were calculated (Kohavi \& Provost, 1998).

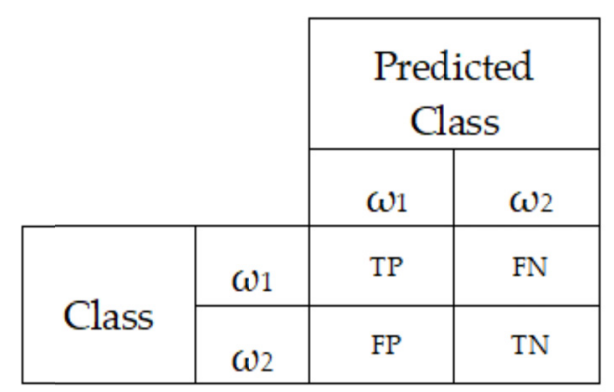

Figure 2. Adaptation of a confusion matrix formation; TP-True positive; FN-False negative; FP-False positive; TN-True negative. Adapted from (Santra \& Christy, 2012)

The Kappa (K) index (Equation 4), which ranges from 0 to 1, provides a measure that is the difference between the concordance examined in the precision of the method employed and the randomized values. In this index, the values are considered excellent when $\mathrm{K}>0.81$ (Cohen, 1960; Landis \& Koch, 1977).

$$
\mathrm{K}=\frac{\sum_{\mathrm{i}=1}^{\mathrm{c}} \omega_{\mathrm{ii}}-\sum_{\mathrm{i}=1}^{\mathrm{c}}\left(\omega_{\mathrm{i}+} \cdot \omega_{+\mathrm{i}}\right)}{\mathrm{n}^{2}-\sum_{\mathrm{i}=1}^{\mathrm{c}}\left(\omega_{\mathrm{i}+} \cdot \omega_{+\mathrm{i}}\right)}
$$

Where, $\omega_{\mathrm{ii}}$ is the total number of correctly sorted samples; $\omega_{\mathrm{i}+}$ is the total number of samples sorted for category i; $\omega_{+\mathrm{i}}$ is the total of samples collected from category $i$; $n$ is the total number of samples; $c$ is the number of categories.

The precision (Equation 5) is the rate of correct predictions performed by the classification model on the dataset, that is, it is the proportion of instances that legitimately belong to a class by the total of cases that is classified into such category.

$$
\operatorname{Pr}=\frac{T p}{T p+F p}
$$


Where, $\mathrm{Tp}$ is the total of the category classified as true positive; Fp is the total of categories classified as false positive.

Sensitivity (Equation 6) is the ratio of true positives, that is, the model ability to perfectly predict the true class.

$$
\mathrm{S}=\frac{\mathrm{Tp}}{\mathrm{Tp}+\mathrm{Fn}}
$$

Where, $\mathrm{Tp}$ is the total of the category classified as true positive; Fn is the total of categories classified as false negative.

The accuracy (Equation 7) is defined as the ratio of correct classifications, without false positives and negatives.

$$
A c=\frac{T p+T n}{T}
$$

Where, Tp is total of the classifier category as true positive; $\mathrm{Tn}$ is the total of the classes categorized as true negative; $T$ is the total dataset.

\section{Results}

The set of seeds used presents several damages, which can be found in the same embryos. For example, some typical patterns can be observed, such as mechanical damage (DM), characterized by abrasions (Figures 3a and $3 \mathrm{~b}$ ); characteristic damages of moisture deterioration (DU), such as intense red or white lesions on the tissues (Figures $3 \mathrm{c}$ to $3 \mathrm{k}$ ); whitish circular lesions, typical of bedbug bites (DP) (Figure 3k to 3o), and also embryos without apparent lesions (SL) (Figure 3p).

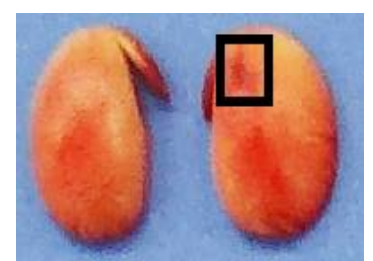

a) $\mathrm{DM}$

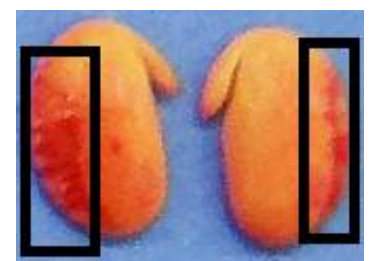

e) $\mathrm{DU}$

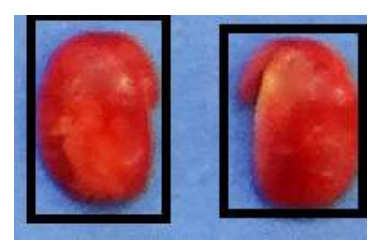

i) $\mathrm{DU}$

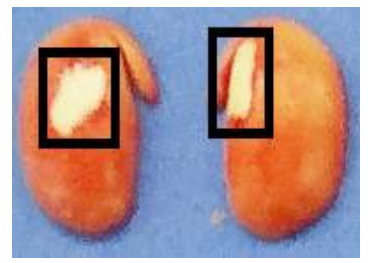

m) $\mathrm{DP}$

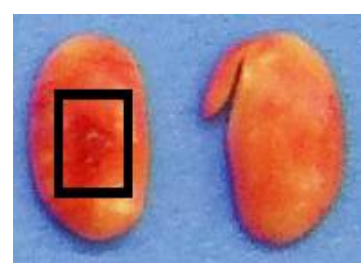

b) $\mathrm{DM}$

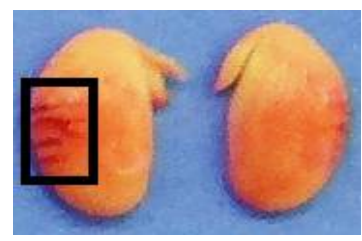

f) $\mathrm{DU}$

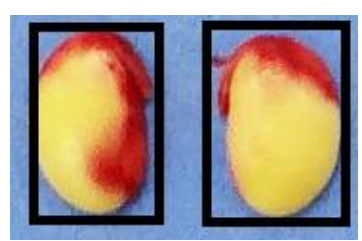

j) $\mathrm{DU}$

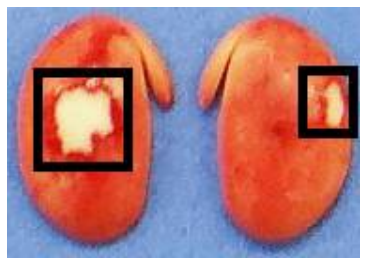

n) $\mathrm{DP}$

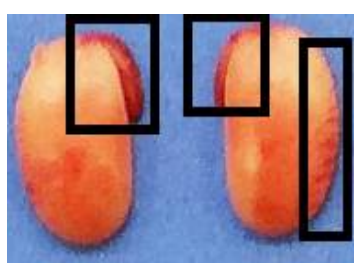

c) $\mathrm{DU}$

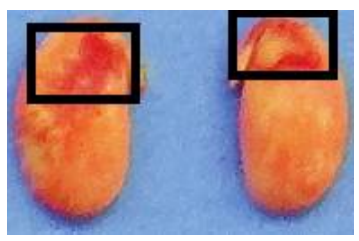

g) $\mathrm{DU}$

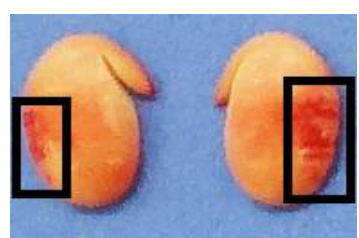

k) $\mathrm{DU}$

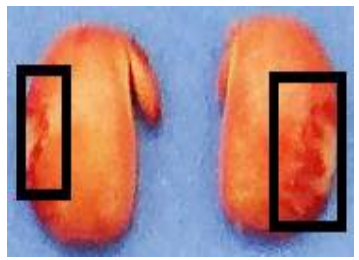

o) $\mathrm{DP}$

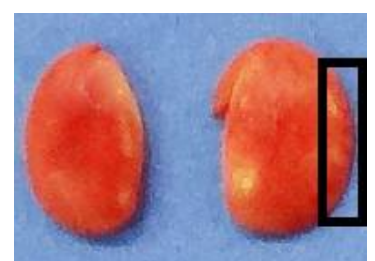

d) $\mathrm{DU}$

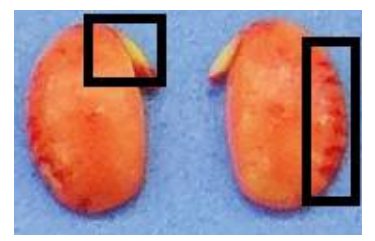

h) $\mathrm{DU}$

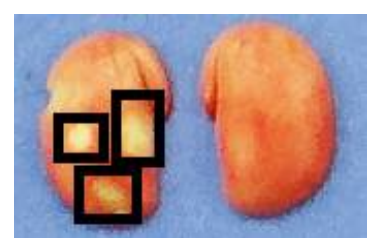

1) $\mathrm{DP}$

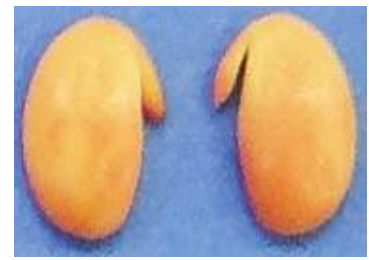

p) $\mathrm{SL}$

Figure 3. Example of damage present in the training set; MD-Mechanical damage, DH-Deterioration by humidity, BD-Bed bug damage, ND-No damage 
The image that shows the training set (Figure 4) was obtained from the composition of an image showing 16 soybean embryos, selected from the images of replication I and II, which satisfactorily describe the standards contained in the tetrazolium test, covering mechanical damage, moisture, bed bugs, and healthy embryos.

Through the interface of extraction of characteristics and training of the classifier model, we identified in the training images the regions with the background patterns (marked in gray), bedbug damage (marked in green), deterioration due to humidity (marked in cyan), and healthy embryos (marked in yellow) (Figure 4). In the process, 39 regions belonging to the background class of the image (68,236 pixels) were detected, also, 141 regions were classified as no damage (48,005 pixels), 2 regions were classified as mechanical damage (470 pixels), 59 regions were classified as deterioration by humidity (30,354 Pixels), and 8 regions were classified as bedbug damage (2,775 pixels). They create a training dataset with 143,291 instances to describe the classes.

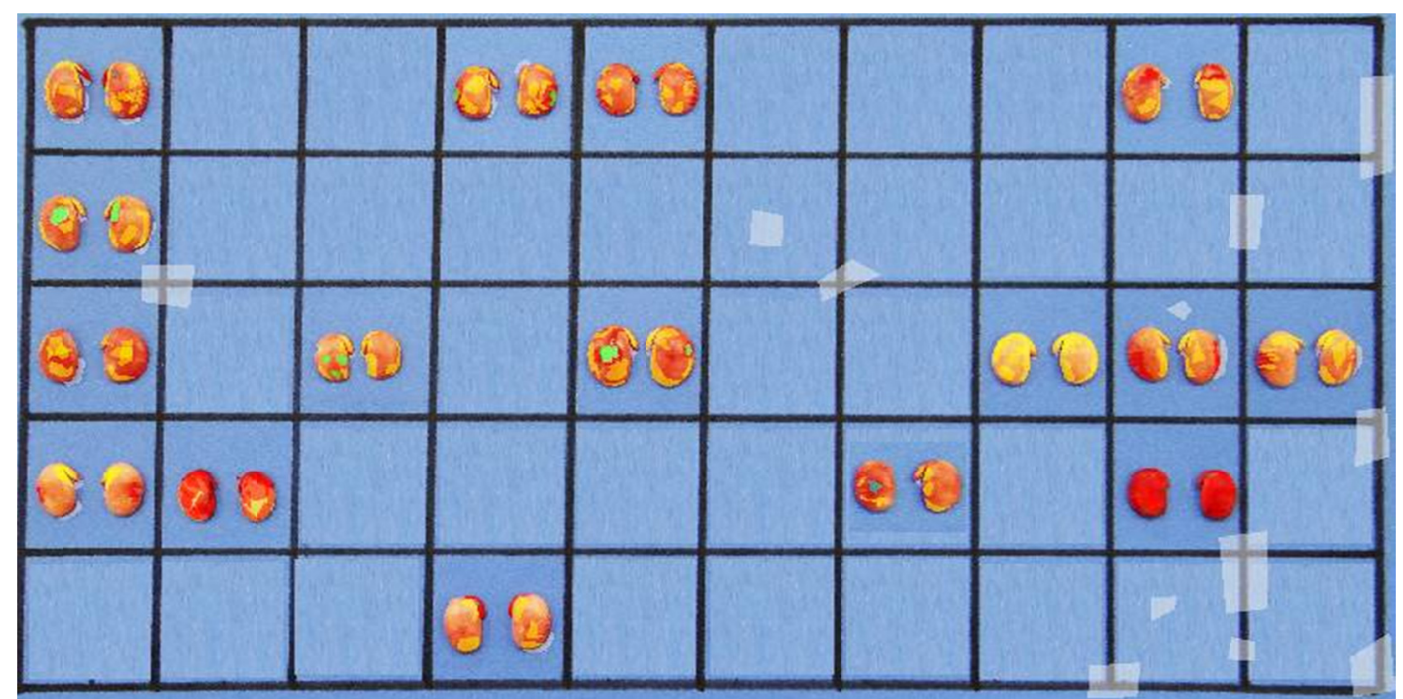

Figure 4. Regions belonging to each class

Once the regions of interest were delimited, the classifier training was performed and, as a result, the classified training image was obtained (Figure 5).
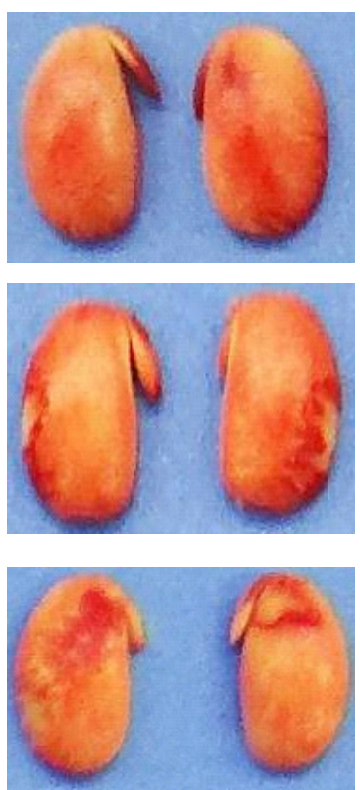
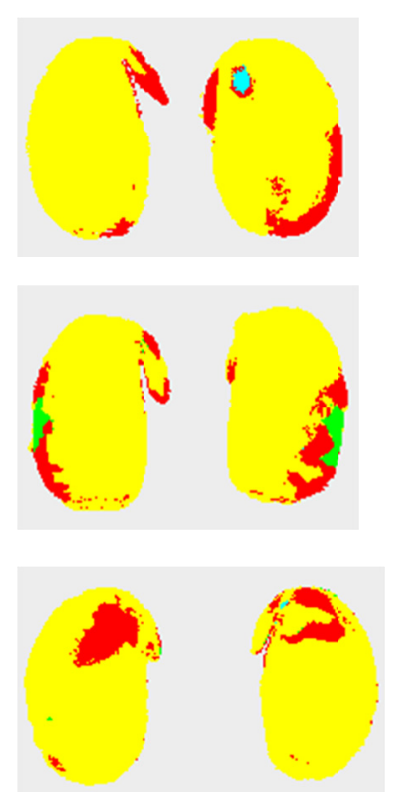
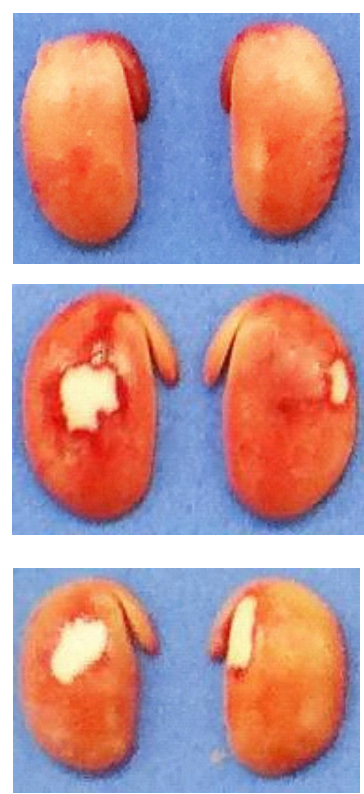
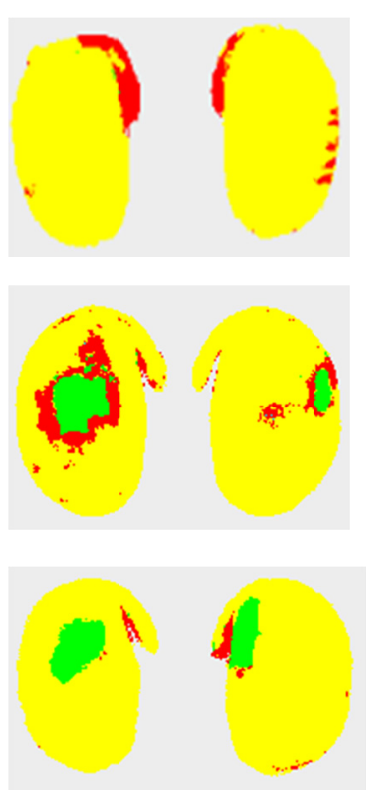

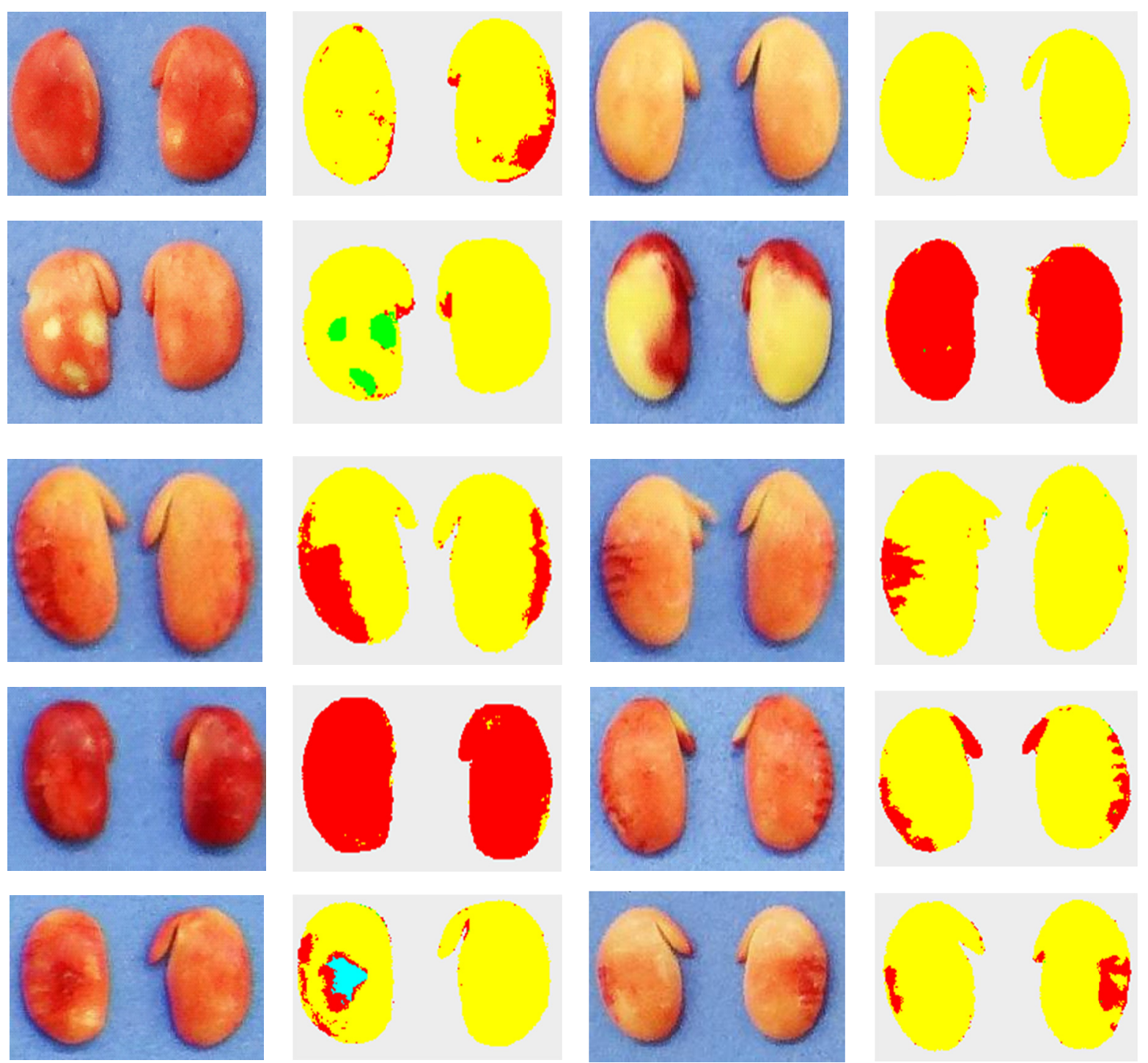

Figure 5. Training images and their sectioned classification; Color legend for segmented images: Gray-background; Red-deterioration by humidity; Green-bed bug damage; Cyan-mechanical damage; Yellow-vigorous tissue

After the image analysis, we realized that a model that would adequately classify the patterns contained in the training image was obtained. This model was the study object to test its own performance.

Table 1 presents the confusion matrix generated from the results obtained by the classifier for the classification data set. 
Table 1. Confusion matrix

\begin{tabular}{lllllll}
\hline \multirow{2}{*}{ Class } & \multicolumn{5}{c}{ Predicted class } & \multirow{2}{*}{$\boldsymbol{\text { B }}$} \\
\cline { 2 - 6 } & Background & ND & MD & DH & BD & 79.459 \\
B & 79.255 & 80 & 0 & 121 & 3 & 90.843 \\
MD & 119 & 89.019 & 32 & 1.592 & 81 & 1.718 \\
DH & 1 & 174 & 1.060 & 483 & 0 & 52.290 \\
BD & 201 & 3.421 & 60 & 48.531 & 77 & 6.078 \\
$\Sigma$ & 16 & 794 & 0 & 487 & 4.781 & 230.388 \\
\hline
\end{tabular}

Note. B-Background; ND-No-damage; MD-Mechanical damage; DH-Deterioration by humidity; BD-Bed bug damage.

We observed that 80 instances among the 79,459 instances of the background class were erroneously classified as healthy embryos, 121, as deterioration by moisture, and three, as bedbug damage. Therefore, the accuracy of the class was $99.7 \%$, with sensitivity of $99.7 \%$ and precision of $99.6 \%$.

Regarding no-damage class, 119 instances among 90,843 were misclassified as background, 32 as mechanical damage, 1,592 as deterioration by moisture, and 81 as bedbug damage. The accuracy was $98 \%$, with sensitivity of $96.6 \%$, and accuracy of $95.2 \%$. The mechanical damage class was the one with the lowest correctness, since, among 1,718 instances, one instance was wrongly classified as background, 174 as healthy embryos, and 483 as deterioration by humidity. Its accuracy was $61.7 \%$, with sensitivity of $73.9 \%$, and precision of $92 \%$.

The class of deterioration by humidity presented 201 instances classified as background, 3,421 classified as undamaged, 60 as mechanical damage, and 77 as bedbug damage, obtaining accuracy of $92.8 \%$, sensitivity of $93.8 \%$ and precision of $95.2 \%$. Also, the class of bedbug damage presented 16 instances that were mistakenly classified as background, 794 as no-damage, and 487 as deterioration by humidity. Its accuracy was 78.7 , with sensitivity of $86.8 \%$, and precision of $96.7 \%$.

The accuracy, sensitivity, precision, and Kappa index values for the model were calculated in relation to data, based on the values of the confusion matrix and cross-validation (Table 2).

Table 2. Classifier performance results

\begin{tabular}{lllllll}
\hline & Ac (\%) & Pr (\%) & S (\%) & Icc (\%) & Iic (\%) & K \\
\hline B & 99.7 & 99.6 & 99.7 & & & \\
ND & 98 & 95.2 & 98 & & & \\
MD & 61.7 & 92 & 61.7 & & & \\
DH & 92.8 & 94.8 & 92.8 & 96.6 & & \\
BD & 78.7 & 96.7 & 78.7 & & \\
$\mathrm{x}_{\mathrm{p}}$ & 96.6 & 96.6 & 96.6 & & & \\
\hline
\end{tabular}

Note. B-Background; ND-No-damage; MD-Mechanical damage; DH-Deterioration by Humidity; BD-Bed bug damage; Ac-Accuracy; Pr.-Precision; S-Sensitivity; Icc-Instances correctly classified; Iic-Instances incorrectly classified; K-Kappa Index; $\mathrm{x}_{\mathrm{p}}$-Weighted average.

By the analysis of the results, we observed that, 222,646 instances among 230,388 instances were correctly classified (96.7\%), and 7,742 were incorrectly classified (3.4\%), with a Kappa index of 0.95 . Kulkarni and Lowe (2016), reached similar results studying RF algorithm for land cover classification concluded that their performance was better than all other studied classifiers in terms of overall accuracy and kappa coefficient. Chan and Paelinck (2008), evaluating Random Forest and Adaboost classification for ecotope mapping using hyperspectral imagery concluted that in terms of accuracy performance, RF have outperformed a neural network classifier.

\section{Conclusion}

Supervised classification combined with pixel-based segmentation has proved to be efficient at extracting information from the tetrazolium test, allowing more accurate evaluations with less subjectivity. Moreover, the image attribute choices, along with the Random Forests algorithm, were efficient in the process of sample 
classification with high dimensionality, which leads the development of new alternatives technologies facilitating to perform exhaustive visual tests.

\section{Acknowledgements}

Authors are thankful to Western Paraná State University, Federal University of Technology-Paraná and Coordination for the Improvement of Higher Education Personnel/Araucária Foundation (CAPES/FA) for supporting this research.

\section{References}

Akar, Ö., \& Güngör, O. (2012). Classifcation of Multispectral Images Using Random Forest Algorithm. Journal of Geodesy and Geoinformation, 1(2), 06-112. https://doi.org/10.9733/jgg.241212.1

Andrade, A. C. de, Francisco, C. N., \& Almeida, C. M. (2015). Desempenho de Classificadores Paramétrico e Não Paramétrico Na Classificação da Fisionomia Vegetal. Anais XVII Simpósio Brasileiro de Sensoriamento Remoto, João Pessoa, Brazil.

Arganda-Carreras, I., Cardona, A., Kaynig, V., Rueden, C., \& Schindelin, J. (2011). Trainable WEKA segmentation. Retrieved from https://imagej.net/Trainable_Weka_Segmentation

Arganda-Carreras, I., Kaynig, V., Rueden, C., Schindelin, J., Cardona, A., \& Seung, H. S. (2016). Trainable_Segmentation: Release v3.1.2.

AOSA (Association of Official Seed Analysts). (1983). Seed vigor testing handbook. Ithaca, NY, USA.

Awcock, G. J., \& Thomas, R. (1996). Applied Image Processing. Publisher: Mcgraw-Hill, New York, EUA. https://doi.org/10.1007/978-1-349-13049-8

Breiman, L. (1996). Bagging Predictors. Machine Learning, 24(2), 123-140. https://doi.org/10.1023/A:101805 4314350

Breiman, L. (2001). Random Forests. Machine Learning, 45(1), 5-32. https://doi.org/10.1023/A:1010933404324

Chan, J. C., \& Paelinckx, D. (2008). Evaluation of Random Forest and Adaboost tree-based ensemble classification and spectral band selection for ecotope mapping using airborne hyperspectral imagery. Remote Sensing of Environment, 112(6), 2999-3011. https://doi.org/10.1016/j.rse.2008.02.011

Cohen, J. A. (1960). Coefficient of Agreement for Nominal Scales. Educational and Psychological Measurement, 20(1), 37-46. https://doi.org/10.1177/001316446002000104

Dias, V. R. M., Sanches, L., Alves, M. C., \& Nogueira, J. S. (2012). Spatio-Temporal Variability of Anions in Wet Precipitation of Cuiabá, Brazil. Atmospheric Research, 107, 9-19. https://doi.org/10.1016/j.atmosres. 2011.11.003

França Neto, J. B., \& Krzyzanowski, F. C. (2018). Metodologia do teste de tetrazólio em sementes de soja. Londrina, PR: EMBRAPA-CNPSo.

França Neto, J. B., Krzyzanowski, F. C., Henning, A. A., \& Costa, N. P. (2000). Tecnologia de Produção de Sementes-A Cultura da Soja No Brasil (pp. 117-123). Embrapa Soja, Brazil.

Geisser, S. (1993). Predictive Inference. Publisher: Chapman and Hall, New York, EUA. https://doi.org/ 10.1007/978-1-4899-4467-2

Goldstein, B. A., Polley E. C., \& Briggs, F. B. (2011). Random Forests for Genetic Association Studies. Statistical Applications in Genetics and Molecular Biology, 10(1), 32. https://doi.org/10.2202/15446115.1691

Hall, M., Frank, E., Holmes, G., Pfahringer, B., Reutemann, P., \& Witten, I. H. (2009). The Weka Data Mining Software: An Update. Acm Sigkdd Explorations Newsletter, 11(1). https://doi.org/10.1145/1656274. 1656278

Huang, J., Siu, \& Liu, W. (2015). Fast Image Interpolation via Random Forests. IEEE Transactions on Image Processing, 24(10), 3232-3245. https://doi.org/10.1109/TIP.2015.2440751

Kohavi, R., \& Provost, F. (1998). Glossary of Terms. Machine Learning, 30, 271-274. https://doi.org/10.1023/ A:1017181826899

Kulkarni, A. D., \& Lowe, B. (2016). Random Forest Algorithm for Land Cover Classification. Computer Science Faculty Publications and Presentations, 4(3), 58-63. http://hdl.handle.net/10950/341 
Landis, J. R., \& Koch, G. G. (1977). The Measurement of Observer Agreement for Categorical Data. Biometrics, 33(1), 159-174. https://doi.org/10.2307/2529310

Liaw, A., \& Wiener, M. (2002). Classification and Regression by Random Forest. $R$ News, 2(3), 18-22.

Marcos Filho, J. (2015). Fisiologia de Sementes de Plantas Cultivadas. Embrapa Soja (CNPSO), Londrina, Brazil.

Moore, R. P. (1985). Handbook on Tetrazolium Testing. International Seed Testing Association, Zurich, Switzerland.

Nguyen, C., Wang, Y., \& Nguyen, H. N. (2013). Random Forest Classifier Combined with Feature Selection for Breast Cancer Diagnosis and Prognostic. J. Biomedical Science and Engineering, 6(5), 551-560. https://doi.org/10.4236/jbise.2013.65070

Santra, A. K., \& Christy, C. J. (2012). Genetic algorithm and confusion matrix for document clustering. International Journal of Computer Science Issues, 9(1), 322.

Schindelin, J., Arganda-Carreras, I., Frise, E., Kaynig, V., Longair, M., Pietzsch, T., ... Cardona, A. (2012). Fiji: An open-source platform for biological-image analysis. Nature Methods, 9(7), 676-682. https://doi.org/ 10.1038/nmeth.2019

Schindelin, J., Rueden, C. T., Hiner, M. C., \& Eliceiri, K. W. (2015). The ImageJ ecosystem: An open platform for biomedical image analysis. Molecular Reproduction and Development, 82(7), 518-529. https://doi.org/ $10.1002 / \mathrm{mrd} .22489$

\section{Copyrights}

Copyright for this article is retained by the author(s), with first publication rights granted to the journal.

This is an open-access article distributed under the terms and conditions of the Creative Commons Attribution license (http://creativecommons.org/licenses/by/4.0/). 